\title{
Organocatalytic Carbonyl-Olefin Metathesis Using a Hydrazine Catalyst
}
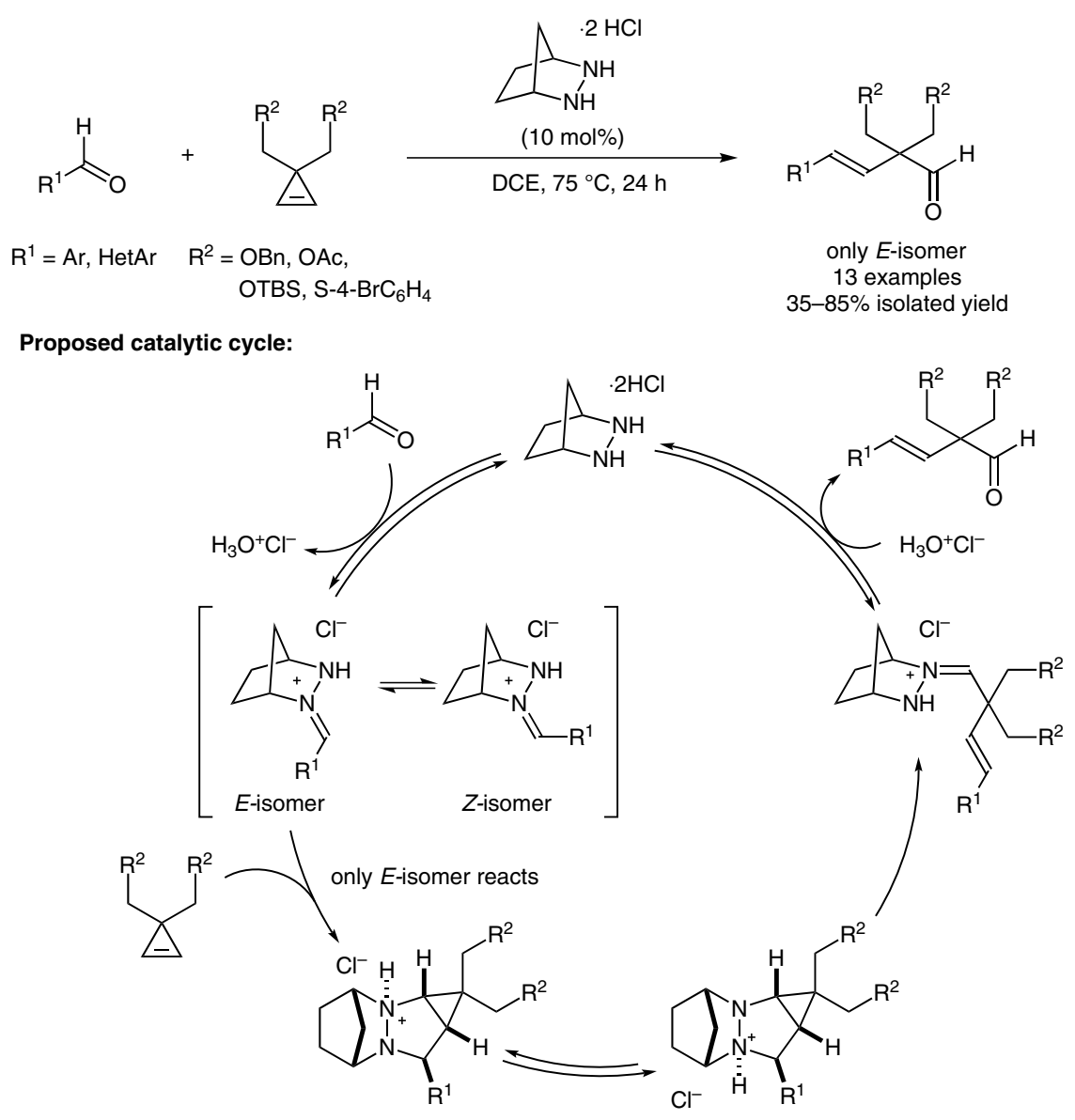

Key words

carbonyl-olefin metathesis

hydrazines

cycloaddition

cycloreversion

Significance: Lambert and co-workers have developed a carbonyl-olefin metathesis catalyzed by a bicyclic hydrazine. The reaction is based on the idea to replace the typically used [2+2]-cycloaddition-cycloreversion approach with a [3+2] analogue, which is thermochemically allowed. The reaction was found to require a bicyclic hydrazine as the bishydrochloride in order to proceed and is essentially restricted to aromatic aldehydes due to the many degradation pathways for aliphatic aldehydes in the presence of the hydrazine catalyst. The authors propose a catalytic cycle, in which the observed $E$-selectivity is explained.
Comment: Metathesis reactions involving carbonyls are much less developed than reactions of olefins and alkynes with themselves. This work by Lambert and co-workers will hopefully encourage further developments in this field. Future challenges are the use of unactivated acyclic olefins, leading to a fragment coupling reaction, and the development of a desymmetrization of mesocyclopropenes using an enantiomerically pure hydrazine catalyst or by replacing the chloride counteranion with one of the recently established chiral counteranions, such as BINOL-derived phosphates.

SYNFACTS Contributors: Benjamin List, Manuel Mahlau 